\title{
Optimization of Horizontal Current in Metal Pad by using Slotted Cathode and Heightened Collector Bars
}

\author{
Kaiyu Zhang ${ }^{1, a}$ and Yuqing Feng ${ }^{2, b}$ \\ ${ }^{1}$ Department of mechanical engineering, Hefei University of Technology, Hefei, 230000, China \\ ${ }^{2}$ CSIRO Computational Informatics, Box 312, Clayton South, VIC 3169, Australia \\ akaiyuzhang@hfut.edu.cn, ${ }^{\text {b}}$ Yuqing.Feng@csiro.au
}

Keywords: Aluminium electrolytic, Horizontal current, Optimal design, Numerical modelling.

\begin{abstract}
According to the problem that horizontal current in the metal pad of aluminum reduction cell induces waves of the electrolyte-metal interface, this paper reports on assessments of the horizontal current elimination effects by using slotted cathode and heightened collector bar as well as their combination. Computational fluid dynamics (CFD) modelling was applied to predict the current flow and the corresponding voltage drop across the electrolytic cell. The results show that both appropriate design schemes of slotted cathode and heightened collector bars can effectively reduce the maximum and average values of horizontal current density in the metal pad. Additionally, their combination effect of horizontal current reduction is better than each of both.
\end{abstract}

\section{Introduction}

The Hall-Héroult cell is the main equipment at the industrial scale for producing aluminum from alumina. It is actually an electrochemical cell where alumina is dissolved in the molten electrolyte and then electrolyzed. The direct electric current is fed between the anodes and an underlying cathode, to cause electrochemical reduction of alumina reactant to aluminum which settles onto a liquid metal pool lying over the cathode, as show in Fig. 1.

In recent decades, the scale of the commercial cells has been substantially enlarged gradually, so does the working current (300-500KA). The huge amount of electricity flow induces vast magnetic field. The coupling effect of horizontal electricity-vertical magnetic field causes electromagnetic force (Lorentz force). This Lorentz force acting on the bath-metal field influences the global scale flow of the cell. It introduces the metal pad motion, waves into the bath-metal interface, and indirectly results in voltage fluctuations, and high local current density. These effects would decrease the working stability and the current efficiency of the cell. In worst case, the stirring metal contacts and impacts with the anode which lead to the reoxidation of the aluminum. Therefore, reducing the horizontal current and predicting the resulting electromagnetic force is important to quantitatively assess its effect on cell performance [1].

Be cognizant of the adverse effects of the horizontal current, some researches have been focused on the causes of it, and try to minimize it. It was found out that the principal thing which strongly influences the current is the electrical conductivity of the materials within the cell: the aluminum is an excellent conductor, the carbon a moderate conductor, and the cryolite a very poor conductor. This ordering of the conductivities is critical to the way in which the current distributes within the cell, because the current would flow through the least electricity resistance automatically [2]. Since the physical property of these materials and the overall design structure of the Hall-Héroult cell cannot be greatly changed, adjusting and modifying the features of the cathode or the collector bar have been proposed to optimize the current direction.

Feng [3] proposed that using the cathode with an inclined surface, the horizontal current can be greatly decreased even eliminated. Winttner [4] put forward a type of cathode carbon block with higher resistivity near the side of cell to rearrange current path. Wenju [5] designed a type of cathode carbon block with a horizontal slot (filled with refractory cement), acting as an insulator to delimit the 
electricity passage. Yang [6] tried to optimize the current direction with a design scheme of heightened collector bars.

All these literatures claimed that their designs have a remarkable effect on horizontal current reduction. However, there is no study compares these designs in terms of application on the same cell. Moreover, each literature proposed only one design either of the cathode or the collector bars. No research has been conducted to study the possible results of the combinations of these design schemes.

In this work, the horizontal current elimination effects of the slotted cathode carbon design and the ladder shaped collector bar was compared by using the finite control volume method. Additionally, the coupling effects of the two designs was also investigated.

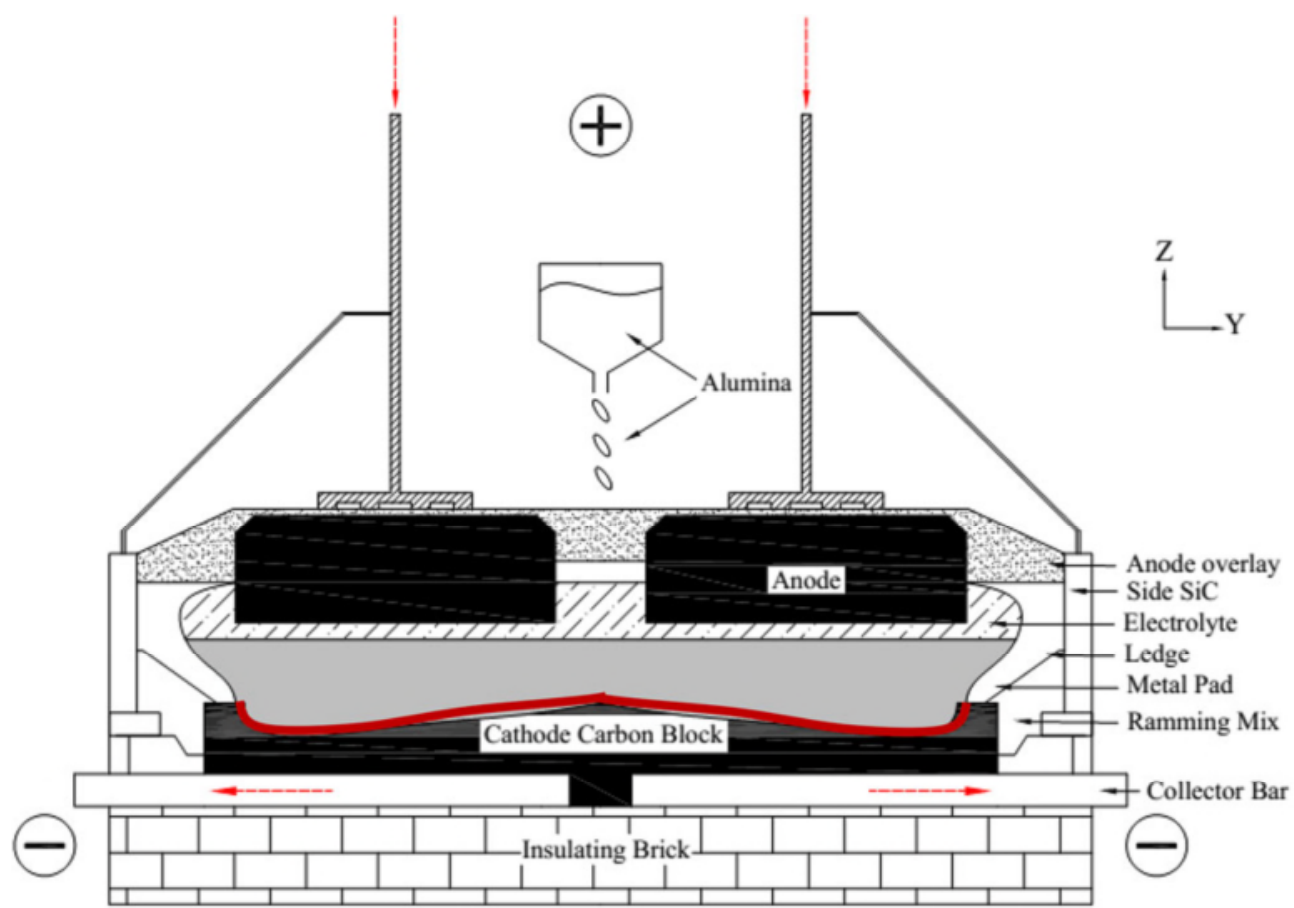

Fig. 1 The schematic representation of a Hall-Héroult cell.

\section{Analysis Model and Theory}

Electrical current flow can be simulated by using different modelling methods and numerical platforms, such as finite element analysis (FEA) modelling (Abaqus and ANSYS Mechanical APDL) and finite control volume method in CFD modelling (ANSYS CFX and Fluent). The FEA model treats every part as a solid, but it is not possible to simulate the dynamic motion of bubbles planned for future work. The ANSYS/Fluent software has been used in the past to study bubble dynamics [7], and has the potential for fully coupling bubble flow and current flow in future work, and is therefore, selected for this study.

For a fast analysis and considering the symmetrical design of the cell, this study is conducted using a two-dimensional (2D) geometry. Fig. 2 shows the 2D geometry used in the simulation. The geometry represents a slice of a typical conventional commercial 300-KA Hall-Héroult prebake cell, but is not related to any specific cell design. To reasonably represent a real aluminium smelting process, the parts included in the model are: anode, electrolyte, metal, cathode and collect bar (Fig. 2a). Fig. 2b shows the geometry model of a cathode structure with a slotted cathode. The slot formed in the cathode carbon block is filled with insulating board. Fig. 2c shows the geometry model of a heightened collector with corresponding deformed cathode. Fig. $2 \mathrm{~d}$ shows the combination design of both.

Current flows in from the top of the anode and flows out from the end of the collect bar, thus these two faces are set as an inlet and outlet for the current, respectively. Besides left side is set as symmetry boundary condition, all the other boundaries are set as insulation boundaries. At the top surface of the 
anode, a constant current density of $1 \mathrm{~A} / \mathrm{cm}^{2}$ is assigned. At the right end of the collect bar where the current flows out, the value of voltage is set to zero. This setting is convenient as it allows the whole voltage drop of the full cell based on the voltage value at the anode top surface to be calculated.

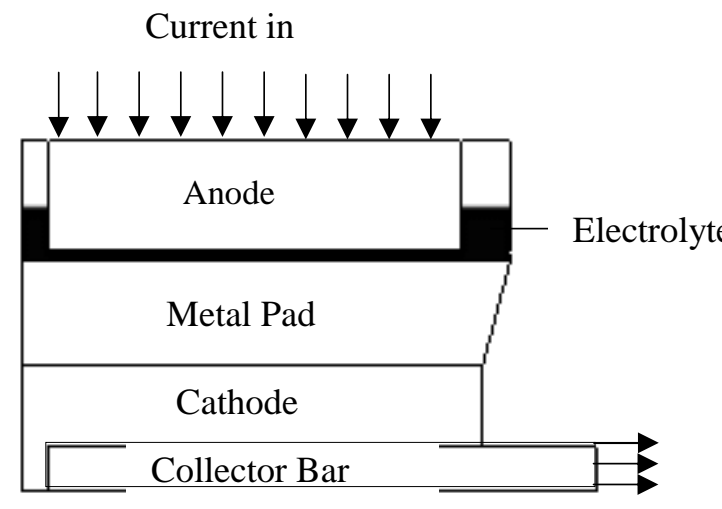

Current out

(a) Case A: Conventional Hall-Héroult Cell Structure

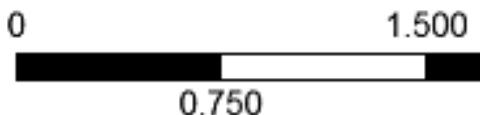

0.750

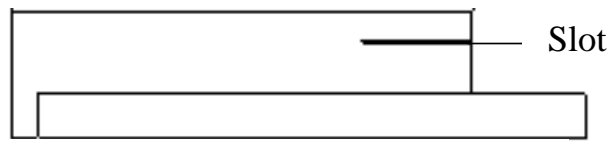

(b) Case B: Slotted Cathode

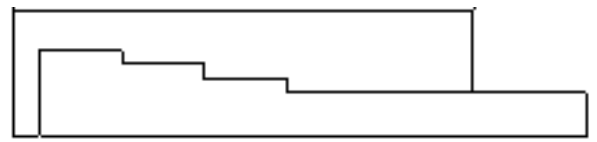

(c) Case C: Heightened Collector Bar

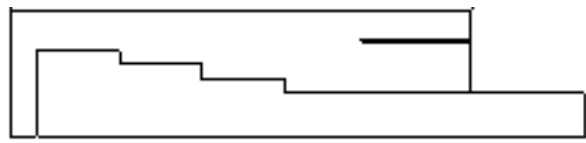

(d) Case D: Combination of Slotted Cathode and Heightened Collector Bar

2.250

Fig. 2 The schematic representation of a Hall-Héroult cell.

To calculate electrical redistribution induced by the modified structure of cathode and collect bar, the Laplace equation:

$\nabla(\sigma \nabla \mathrm{V})=0$

is solved within the domain. $\mathrm{V}$ represents electrical potential or voltage and $\mathrm{r}$ is the electrical conductivity, which varies for different materials.

The commercial CFD package ANSYS/Fluent, which is based on the Finite Volume Method, was used as the numerical modelling tool. The longer term plan is to resolve the bath, bubble and metal pad motion while simultaneously predicting the electrical current distribution. Thus, the CFD model with an additional Laplace equation for the electrical field is used in this work. Using such a setup in Fluent requires the Navier-Stokes equation to solve for the bath, gas and metal pad flow. As the present work focuses on predicting the electrical current flow for specific cell structure modification, only the scalar equation (V) needs to be solved.

In setting up the model, the following simplifications are made:

- The bubble in the cell is not included due to the purpose of simplifying the models. It is believed that bubble distribution beneath the anode has a large effect on voltage drop as the current mainly flows through the anode-cathode distance [8]. Therefore, if bubbles are also involved in the models, the effects of slotted cathode and heightened collector bar would be hard to define.

- The effects of temperature and alumina concentration on the electrical conductivity is not considered

- The effect of liquid motion on current flow is neglected;

As showed in Fig. 2, a total of 4 cases were simulated. Case A represents the full geometry condition of the conventional cell, which is used as the baseline to quantify current optimization effects of other modified cell designs. Case B and case C correspond to the design of slotted cathode and heightened collector bar separately. Because the other parts of the cell have not changed, only structures of the improved cathode and collector bar are shown. In the model, the effects of the slot size, slot position and extra height of collector bar were not investigated, because these factors have already been studied $[5,6,9]$. Consequently, according to these studies, the optimum parameters have been selected in current work. 


\section{Results and Discussion}

Fig. 3 shows the vector plot of the electricity distribution in the whole model of 4 cases, indicating that either the slotted cathode or the heightened collector bar significantly changes the current path, so does their combination. The maximum horizontal current density in the conventional cell is $15844 \mathrm{~A} / \mathrm{m}^{2}$ located around the right side of the bath-cathode interface (captured by the red ellipse in Fig. 3a). With slot, the maximum current density shifts from the edge toward the cell center. Moreover, compared with case A, the current path has been greatly improved, more electricity is beginning to lean to a vertical direction within the metal pad. Though the entire current direction is upgraded, the local maximum current is slightly increased to $16984 \mathrm{~A} / \mathrm{m}^{2}$ (captured by the red ellipse in Fig. 3b). By means of the heightened collector bar, as though the whole pattern of the current does not change and the maximum current density is located in the original position, it decreases to 14666 $\mathrm{A} / \mathrm{m}^{2}$ (captured by the red ellipse in Fig. 3bc). Notably, with the combination of cathode slot and heightened cathode, both the global current distribution and the maximum horizontal value are modified. In case $\mathrm{D}$, the horizontal current is greatly diminished and the maximum value decreases to $14630 \mathrm{~A} / \mathrm{m}^{2}$.

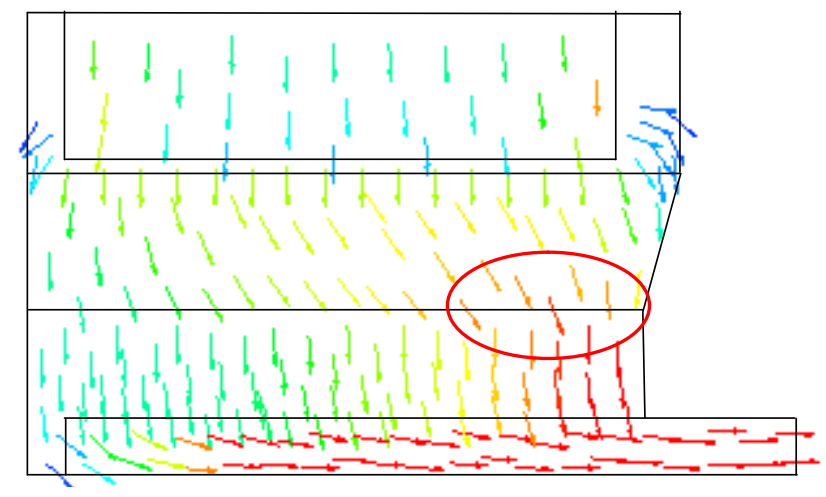

(a) Case A: Conventional Hall-Héroult Cell Structure

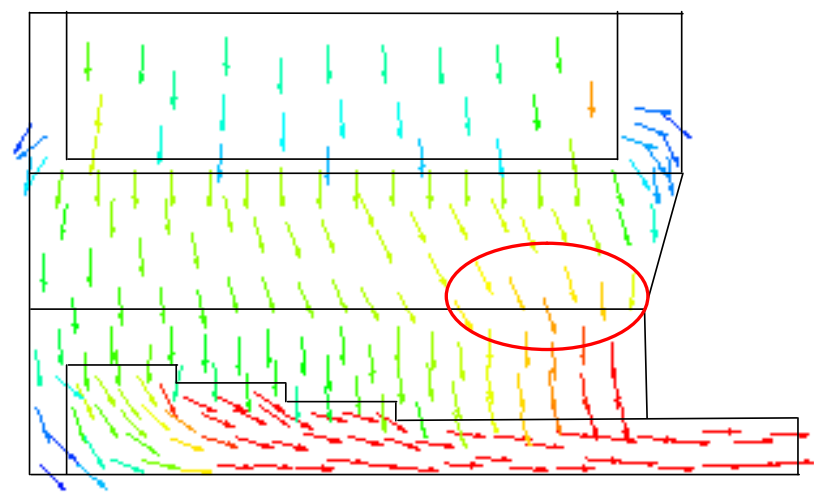

(c) Case C: Heightened Collector Bar Current Density

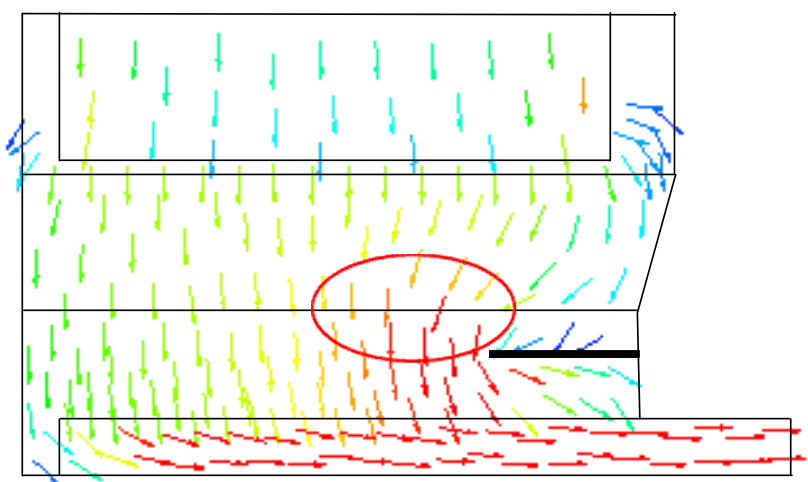

(b) Case B: Slotted Cathode

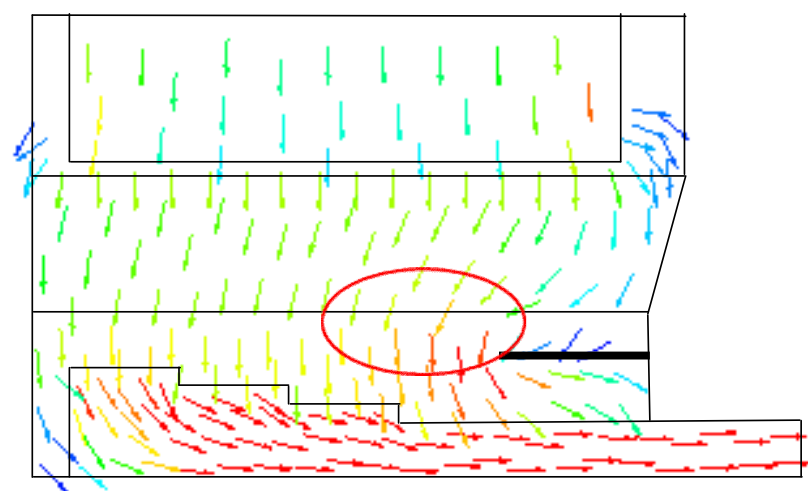

(d) Case D: Combination of Slotted Cathode and Heightened Collector Bar

$\mathbf{A} / \mathbf{m}^{2}$

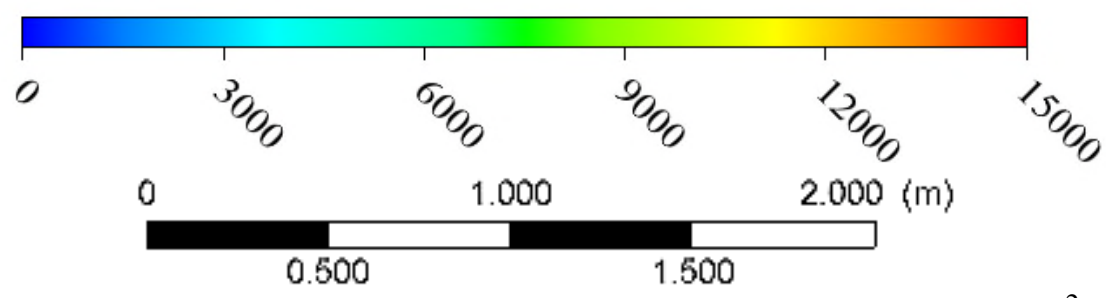

Fig. 3 Vector plot of current density of the cell in $\mathrm{A} / \mathrm{m}^{2}$. 


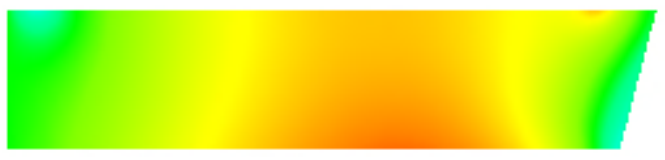

(a) Case A:Conventional Hall-Héroult cell structure

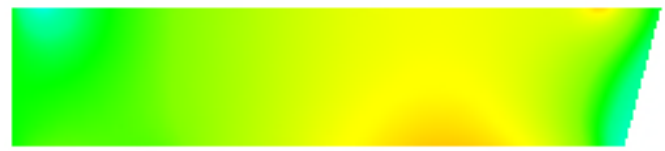

(c) Case C: Heightened Collector Bar

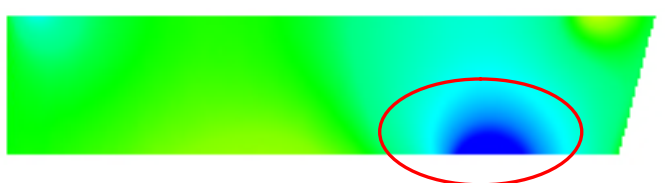

(b) Case B: Slotted Cathode

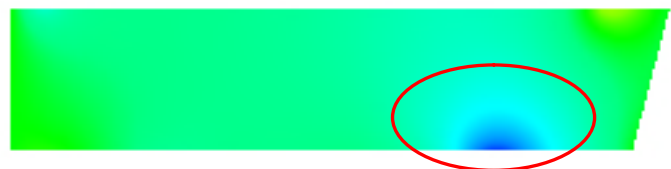

(d) Case D: Combination of Slotted Cathode and Heightened Collector Bar

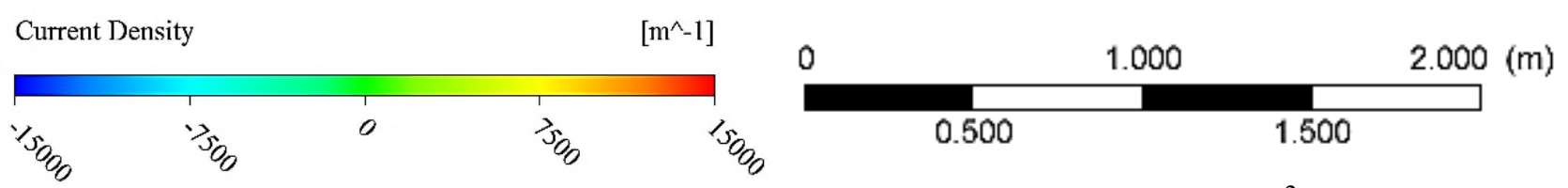

Fig. 4 Contour of horizontal current density in the metal pad in $\mathrm{A} / \mathrm{m}^{2}$.

Fig. 4 shows the contour of horizontal current density distribution in the metal pad. Remarkably, compared with case $\mathrm{A}$, the average horizontal value is considerably reduced by in case $\mathrm{B}$, case $\mathrm{C}$ and case D. Fig. 4 (b) indicates that the slotted cathode can seriously decrease the overall horizontal current, however, results in large negative local current density on the upright position of the inner side of the insulating board (captured by the red ellipse in Fig.4b). It is because the current from upright position of the slot bypasses the insulating board automatically, then reorganize the whole current distribution within the metal pad. Clearly, the heightened collector bar can decrease the whole value of the horizontal current, especially the part of the center part of the metal pad. For the reason that the collector bar's conductivity is much better than the cathode's, the current shifts to the higher position of the collector bar, as a result the horizontal electricity has almost vanished around that area. At the same time, the total horizontal electricity is largely declined. It is worth noting that case 4 which includes the slotted cathode and the heightened collector bar combines both advantages of the designs. Not only does it extensively eliminate the horizontal current but also substantially decreases the local maximum current density.

\section{Conclusion}

Based on the finite control volume method in CFD modelling, the effects of slotted cathode and heightened collector bar as well as their combination on current distribution has been investigated in current work. The conclusions of this study are as follows:

1. The slotted cathode can considerably decrease the overall horizontal current in the metal pad. However, this design induces great negative local current density.

2. The heightened collector bar can also reduce overall horizontal current, especially the center part of the cell. Nevertheless, it only slightly changed the horizontal current of the right part of the metal pad.

3. Advantages of both slotted cathode and the heightened collector bar has been represented in their combination design.

\section{Acknowledgement}

In this paper, the research was sponsored by the Nature Science Foundation of Hefei University of Technology (Project No. XC2015JZBZ13). 


\section{References}

[1] R. J. Moreau and D. Ziegler. Stability of aluminium cells-a new approach, New Orleans, LA: TMS, 1986, 359-364.

[2] P. A. Davidson and R. I. Lindsay. A new model of interfacial waves in aluminum reduction cells, Orlando, FL: TMS, 1997, 437-442.

[3] N.X. Feng, China patent CN201110385777.0 (2012)

[4] H. Wintter and K. Lauer, U.S patent 3,787,311(1974)

[5] W. J. Tao, T. F. Li and Z. W. Wang, et al. Impact of Usage of a Slotted Cathode Carbon Block on Thermoelectric Field in an Aluminium Reduction Cell, The Minerals, Metals Mater. Soc. 5(67) (2015) 929-937.

[6] S. Yang, J. Li and Y. J. Xu, et al. Optimization of Horizontal Current In Metal Pad by Using Cathode with Heightened Collector Bars in Aluminium Reduction Cell. The Chinese J. Nonferr. Metal, 22(10) (2012) 2951-2959.

[7] K. Y. Zhang, Y. Q. Feng and Z. W. Wang, et al. A Numerical Assessment of Bubble-Induced Electric Resistance in Aluminium Electrolytic Cells. J. Appl. Electrochem. 44(10) (2014) 1081-1092.

[8] A. L. Perron, L. I. Kiss and S. Poncsak, et al. The Influence of Bubble Shapeand the Thickness of the Wetting Film on the Incremental Electrical Resistance Caused by the Presence of a Single Bubble in Hall-Heroult Cells. J. Appl. Electrochem. 36 (2006) 1381-1389.

[9] S. Das and Y. Morsi, Geoffrey Brooks. Cathode Characterization with Steel and Copper Collector Bars. JOM, 66(2) (2014) 235-244. 